\title{
A rare clinical presentation of osteomalacia mimicking bony metastatsis in adult
}

Authors:, Nadine Dow, Dr.Anupriya Annapurni, Dr.Vakkat Muraleedharan

Hospital: Kings mill hospital, Sherwood hospital NHS trust, Sutton in Ashfiled

\section{Introduction:}

Vitamin D deficiency in adults results in secondary hyperparathyrodism. This leads to osteoclast activation and bone resorption resulting in osteomalacia, [1] this leads to joint pain and bone shadowing on imaging that may be mistaken for metastatic disease. This case highlights a need for closer monitoring of vitamin D levels especially in elderly patients who may also be suffering from a degree of chronic kidney failure.

\section{Clinical presentation:}

A 74-year-old man was admitted to hospital with diarrhoea, vomiting, abdominal and lower back pain and reduced urine output. Past medical history included hypertension, angina and hypercholesterolemia, chronic kidney disease. Initial diagnosis was campylobacter gastroenteritis and managed accordingly.

Patient developed breathlessness, joint pain, progressive fatigue and persistent microcytic anemia. Gastroscopy and colonoscopy were normal in the past. CT scan of thorax/abdomen/pelvis (figure 1) showed diffuse sclerosis of the spinal vertebrae with lytic areas and extensive patchy lysis of the sacrum and iliac bones, which were thought to be secondary to either bony metastases or metabolic bone disease.

Investigations: Urea 5.6mmol/L (2.9-7.5), Creatinine 134umol/L (70-120), e GFR $48 \mathrm{ml} / \mathrm{min}(>90)$, PTH 336ng/L (14-72), adjusted calcium 2.09mmol/L (2.20-2.60), phosphate 1.01(0.8-1.45), ALP 133U/L (40-130), GGT 12U/L (0-55), 25-hydroxyvitamin D 24nmol/L (51-250), haemoglobin 107g/L (130-180), MCV 81fl (84-102). PSA 2.7mcg/L (0-5), CA 19-9 15 kU/L (0-35), CEA 2 mcg/L (0-2.5) and AFP 2 kU/L (0-10). No serum paraprotein.

Diagnosed as Secondary hyperparathyroidism due to osteomalacia, exacerbated by chronic kidney disease. He was treated with calcium suppliments and cholecalcifoerol 20,000 units weekly for 6-7 weeks followed by 1,800 units weekly. Dexa scan showed osteopenia of the femoral neck.

Six months later, patient was symptamatically as well as biochemically better (table1). Investigations: 25-hydroxyvitamin D 77nmol/L, Adjusted calcium $2.41 \mathrm{mmol} / \mathrm{L}$, urea 11.2, creatinine $189 \mathrm{umol} / \mathrm{L}$, eGFR 32ml/min, PTH 157ng/L. Repeat CT scan(figure2) showed significantly reduced bone sclerosis and little evidence of metabolic bone disease.
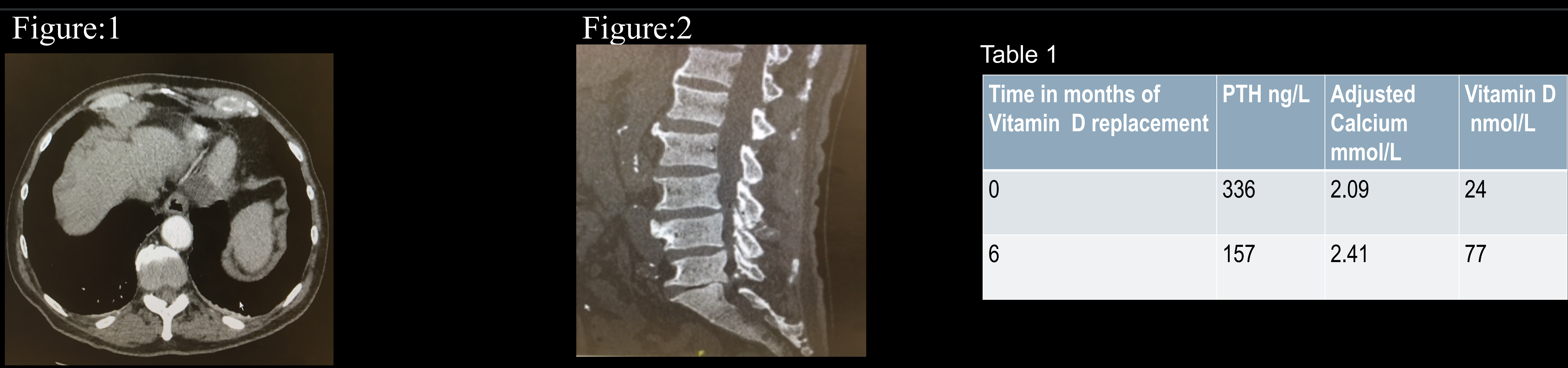

\section{Conclusions:}

Whilst patients with end stage renal failure receiving dialysis are identified as an at risk group for secondary hyperparathyroidism,[2, 3], malabsorption and vitamin D deficiency are other etiologies. Due to the non-specific nature of the symptoms, a severe Vitamin D deficiency can remain undiagnosed for many years resulting in severe clinical manifestations before the patients receive treatment[4]. Elderly housebound patients may have limited sunlight exposure, in addition they may be more at risk of concurrent kidney failure. [6] The inability to convert 25hydroxyvitamin $D$ to the active 1,25-dihydroxyvitamin $D_{3}$ can result not only from kidney failure but also due to decline with normal ageing.[7] Hence these patients are placed at high risk of Vitamin D deficiency, osteomalacia, secondary hyperparathyroidism, bone sclerosis and resulting fractures.[8] Metabolic bone disease should be a differential particularly in this cohort of patients especially the imaging characteristics can mimic metastatic bone disease.

\section{References:}

Holick, M.F., Vitamin D deficiciency. New England Journal of Medicine, 2007. 357(3): p. 266-81.
Salem, M.M., Hyperparathyroidism in the hemodialysis population: a survey of 612 patients. American Journal of Kidney Diseases, 1997. 29(6): p. 862-5.

Salem, M.M. Hyperparathyroidism in the hemodialysis population: a survey of 612 pattients. American Journal of Kidney Diseases, 1997. 29(6): p. 862-5.
Billa, V., et al. High prevalence of hyperparathyroidism among peritoneal dialysis patients: a review of 176 patients. Peritoneal Dialysis International, 2000. 20(3): p. $315-21$.

Key words: PTH- parathyroid hormone, ALP-Alkaline phosphatase,GGT- gamma glutamyl transferase, CEA-carcino embryonic antigen, PSA- prostate specific antigen,AFP-alfa fetoprotein, CT-computed tomography 